\title{
POLYNOMIALLY BOUNDED MULTISEQUENCES AND ANALYTIC CONTINUATION
}

\author{
by DANIEL J. TROY
}

(Received 23 May, 1980)

Given a polynomially bounded multisequence $\left\{f_{m}\right\}$, where $m=\left(m_{1}, \ldots, m_{k}\right) \in \mathbb{Z}_{k}$, we will consider $2^{k}$ power series in $\exp \left(i z_{1}\right), \ldots, \exp \left(i z_{k}\right)$, each representing a holomorphic function within its domain of convergence. We will consider this same multisequence as a linear functional on a class of functions defined on the $k$-dimensional torus by a Fourier series, $\sum g_{m} e^{i m x}$, with the proper convergence criteria. We shall discuss the relationships that exist between the linear functional properties of the multisequence and the analytic continuation of the holomorphic functions. With this approach we show that a necessary and sufficient condition that the multisequence be given by a polynomial is that each of the power series represents, up to a unit factor, the same function that is entire in the variables

$$
\left(1-\exp \left(i z_{1}\right)\right)^{-1}, \ldots,\left(1-\exp \left(i z_{k}\right)\right)^{-1}
$$

1. Notation and terminology. Let $p$ and $k$ be integers, $p \geq 0, k>0$. Let $T_{k}$ be the $k$-dimensional torus and $\mathbb{Z}_{k}$ the collection of $k$-tuples of integers. If $m$ is in $\mathbb{Z}_{k}$ and $x$ is in $T_{k}$, define

$$
\begin{aligned}
& m x=m_{1} x_{1}+\ldots+m_{k} x_{k}, \\
& |m|=\left(m_{1}^{2}+\ldots+m_{k}^{2}\right)^{1 / 2},
\end{aligned}
$$

and

$$
\|m\|=\left|m_{1}\right|+\ldots+\left|m_{k}\right| .
$$

Let $K_{\mathrm{pk}}$ denote the vector space of multisequences $\left\{f_{m}\right\}\left(m \in \mathbb{Z}_{k}\right)$ such that $\left|f_{m}\right||m|^{-2 p}$ is bounded. Let $A_{p k}$ denote the normed linear space of functions defined on $T_{k}$ whose Laplacians of order $p$ exist and have absolutely convergent Fourier series. If $\sum g_{m} e^{i m x}$ is the Fourier series of $g$ in $A_{p k}$, define

$$
\|g\|_{2 p}=\max \left\{\left|g_{0}\right|, \sum|m|^{2 p}\left|g_{m}\right|\right\}
$$

and for every $f$ in $K_{p k}$, define $\langle f, g\rangle=\sum f_{m} g_{-m}$.

For an open $E \subset T_{k}$ denote by $A_{p k}(E)$ the subset of members of $A_{p k}$ with support in $E$. Given $f$ in $K_{p k}$, we will say that $f$ is null on $E$ if $\langle f, g\rangle=0$ for every $g$ in $A_{p k}(E)$. $A_{p k}(E)$ is invariant under multiplication by $e^{i n x}\left(n \in \mathbb{Z}_{k}\right) ;\langle f,$.$\rangle is a continuous linear operator on$ $A_{p k}$; hence, $f$ is null on $E$ if, and only if, for every $n$ in $\mathbb{Z}_{k}$ and $g$ in the $\|\cdot\|_{2 p}$ closure of $A_{p k}(E)$,

$$
\sum f_{m} g_{n-m}=0 .
$$

Let $c$ be a non-negative integer not exceeding $2^{k}-1$. For $j=1, \ldots, k$, denote by Glasgow Math. J. 23 (1982) 41-52. 
$((c, j))$ the coefficient of $2^{k-j}$ in the binary expansion of $c$. Let

$$
D_{c k}=\left\{\left(z_{1}, \ldots, z_{k}\right) \in \mathbb{C}_{k}:(-1)^{((c, j))} \operatorname{Im}\left(z_{j}\right)>0, j=1, \ldots, k\right\} .
$$

Let $\sum$ indicate the summation over all $m$ in $\mathbb{Z}_{k}$, let $\sum^{\prime}$ indicate the sum over all $m$ in $\mathbb{Z}_{k}$ except $(0, \ldots, 0)$, and let $\sum_{(m, c, k)}$ (or $\sum_{(c, k)}$ if the omission of $m$ does not cause ambiguity) indicate the sum to be taken over all $k$-tuples $m$ such that $m_{j} \geq 0$ if $((c, j))=0$ and $m_{j}<0$ if $((c, j))=1$. With this notation, given $f$ in $K_{\mathrm{p} k}$, then

$$
\hat{f}_{c}(z)=\sum_{(c, k)} f_{m} \exp (i m z)
$$

defines a function that is holomorphic in $D_{c k}$.

Given $x$ in $T_{k}$, let us say that $x$ is regular for $f$ in $K_{\mathrm{pk}}$, if there exist $(i)$ a $\mathbb{C}_{k}$ neighborhood $V$ of $x$, (ii) a partitioning

$$
c(1,1), \ldots, c(1, t(1)) ; \ldots ; c(h, 1), \ldots, c(h, t(h))
$$

of some permutation of the integers $0,1, \ldots, 2^{k}-1$ into $h$ cells, (iii) for each integer $u, 1 \leq u \leq h$, complex numbers $d(u, j), 1 \leq j \leq t(u)$, such that

$$
d(u, 1)+\ldots+d(u, t(u))=0,
$$

and (iv) a function $H_{u}$ holomorphic on

$$
V \cup D_{c(u, 1) k} \cup \ldots \cup D_{c(u, t(u)) k}
$$

and such that

$$
\hat{f}_{c(u, j)}(z)=d(u, j) H_{u}(z)
$$

for all $z$ in $D_{c(u, j) k}(u=1, \ldots, h, j=1, \ldots, t(u))$.

If $k=1$, the following statements are equivalent:

(A) $x$ is regular for $f$ for every $x$ in $E$.

(B) $f$ is null on $E$.

(C) The $2 p+2$ derivative of

$$
F(x)=\left(f_{0} x^{2 p+2} /(2 p+2) !\right)+(-1)^{p+1} \sum^{\prime} f_{m} m^{-2 p-2} e^{i m x}
$$

exists and vanishes on $E$.

This result is explicitly stated in [2]. The equivalence of (A) and (B) also follows from [1, p. 116].

2. Statements (A) and (B) with $k \geq 2$. We wish to show that for all $k$ the statement (A) implies the statement (B). The reverse implication does follow in some special cases. For example, if the partitioning (3) were into $2^{k-1}$ pairs

$$
\left(c, 2^{k}-1-c\right), \quad c=0, \ldots, 2^{k-1}-1 .
$$


and, for each $c$,

$$
\sum_{(c, k)} f_{m} g_{-m}+\sum_{\left(2^{k-1}-c, k\right)} f_{m} g_{-m}=0,
$$

for every $g$ in $\mathscr{C}^{\infty}(E)$, then (A) would follow by the "edge-of-the-wedge" [7, Theorem B].

The most obvious illustration that (B) does not imply (A) with $k \geq 2$ has $f_{m}=1$ for all $m$ and $E$ any open subset of $T_{k}$ that does not include the origin. Here $f$ is null on $E$; however, (A) cannot hold if $E$ intersects any of the hyperplanes $z_{j}=0$.

THEOREM 1. Let $f$ be in $K_{\mathrm{pk}}$ and $E$ be an open subset of $T_{k}(p \geq 0, k \geq 2)$. If every $x$ in $E$ is regular for $f$, then $f$ is null on $E$.

Proof. Let $g$ be in $A_{p k}(E)$ and $q$ be a real function, non-negative, $\mathscr{C}^{\infty}$, with compact support, such that $\int g=1$. For every $v>0$, define

$$
g_{v}(x)=\int_{T_{k}} g(x-y v) q(y) d y .
$$

For all $v$ sufficiently small, $g_{v}$ has support in E. Further, for all $v, g_{v}$ is in $\mathscr{C}^{\infty}$ and $g$ is the $L_{1}$ limit of $g_{v}$ as $v \rightarrow 0$.

There exists a $y^{\prime}>0$, such that, for all $c \leq 2^{k}-1, \hat{f}_{c}$ is bounded for all $z=x+i y$, where $x$ is in the support of $g$ and $0<\left|y_{i}\right| \leq y^{\prime}, j=1, \ldots, k$. Therefore, writing

$$
z_{c}=\left(x_{1}+i y(-1)^{((c, 1))}, \ldots, x_{k}+i y(-1)^{((c, k))}\right),
$$

we have uniformly with respect to $y$,

$$
\lim _{v \rightarrow 0}\left|\int_{T_{k}} \hat{f}_{c}\left(z_{c}\right) g(x) d x-\int_{T_{k}} \hat{f}_{c}\left(z_{c}\right) g_{v}(x) d x\right|=0 .
$$

By $[7,10.2]$, the second integral has a limit as $y \rightarrow 0$; hence, so does the first.

To evaluate $\langle f, g\rangle$, we write

$$
\begin{aligned}
\langle f, g\rangle & =\sum f_{m} g_{-m}=\sum_{c=0}^{2^{k}-1} \sum_{(c, k)} f_{m} g_{-m} \\
& =\sum_{u=1}^{h} \sum_{j=1}^{t(u)} \lim _{y \rightarrow 0} \sum_{(c(u, j), k)} f_{m} g_{-m} e^{-\|m\| y} \\
& =\sum_{u=1}^{h} \sum_{j=1}^{t(u)} \lim _{y \rightarrow 0} \int_{T_{k}} \hat{f}_{c}\left(z_{c}\right) g(x) d x,
\end{aligned}
$$

with $c=c(u, j)$ in the $(u, j)$ term of the last double sum. In each term,

$$
\hat{f}_{c}\left(z_{c}\right)=d(u, j) H_{u}\left(z_{c}\right) \text {. }
$$


But for each $u$,

$$
\lim _{y \rightarrow 0} \int_{B} H_{u}\left(z_{c}\right) g(x) d x=\int_{B} H_{u}(x) g(x) d x
$$

$B$ being the support of $\mathrm{g}$, and $\sum_{j=1}^{t(u)} d(u, j)=0$. Hence, $\langle f, g\rangle=0 ; f$ is null on $E$.

As mentioned, the converse of Theorem 1 does not hold; however, an "if and only if" statement can be made for some sets.

Theorem 2. Let $E=T_{k-1} \times I$, where $I$ is an open interval in $T_{1}$. A necessary and sufficient condition that $f$ in $K_{\mathrm{pk}}$ be null on $E$ is that for every even integer $c\left(0 \leq c \leq 2^{k}-2\right)$, there exists a function $H_{c}$, holomorphic on $D_{c k} \cup\left(D_{c / 2, k-1} \times I\right) \cup D_{c+1, k}$, such that $H_{c}(z)=$ $\hat{f}_{c}(z)$ in $D_{c k}$ and $H_{c}(z)=-\hat{f}_{c+1}$ in $D_{c+1, k}$.

Proof. Assume $f$ is null on $E$ and let $g(t)=\sum_{r=-\infty}^{\infty} g_{r} e^{\text {irt }}$ be in $A_{p 1}(I)$. Let $c$ be even $\left(0 \leq c \leq 2^{k}-2\right)$, and $w$ be in $D_{c / 2, k-1}$. Define

$$
u\left(t_{1}, \ldots, t_{k}\right)=g\left(t_{k}\right) \prod_{j=1}^{k-1}\left(1-\exp \left(i w_{j}(-1)^{((c, j))}-t_{j}\right)\right)^{-1} .
$$

Since, for $j=1, \ldots, k-1,((c, j))=((c / 2, j))$ and the factor $(-1)^{(c, j,))}$ serves only to keep the real parts of the exponentials in (4) negative, we can simplify the notation by assuming $c=0$; then, for each $j,((c, j))=0$ and $\operatorname{Im}\left(w_{j}\right)>0$. Let $b_{j}=\operatorname{Im}\left(w_{j}\right)$ and $b=$ $\min \left\{b_{j}: 1 \leq j \leq k-1\right\}$. We may now express the right-hand side of $(4)$ in the form $\sum u_{m} e^{i m t}$, where $u_{m}=0$ if $m_{i}<0$ for some $j \leq k-1$, and

$$
u_{m}=g_{m_{k}} \exp \left(i\left(m_{1} w_{1}+\ldots+m_{k-1} w_{k-1}\right)\right)
$$

if $m_{j} \geq 0$ for all positive $j \leq k-1$. In either case

$$
\left|u_{m}\right| \leq\left|g_{m_{k}}\right| \exp \left(-b_{1} m_{1}-\ldots-b_{k-1} m_{k-1}\right) \text {. }
$$

Hence,

$$
\sum|m|^{2 p}\left|u_{m}\right| \leq k^{p} \sum_{r=-\infty}^{\infty}\left|r^{2 p}\right|\left|g_{r}\right|\left(\sum_{n=0}^{\infty} n^{2 p} e^{-n b}\right)^{k-1} .
$$

Therefore, $u$ is in $A_{p k}(E)$.

By hypothesis, therefore, $\langle f, u\rangle=0$; that is,

$$
\sum_{r=-\infty}^{\infty}\left(\sum_{(m, 0, k-1)} f_{(-m, r)} e^{i m w}\right) g_{-r}=0
$$

for every $w$ in $D_{0, k-1}$. But

$$
\left|\sum_{(0, k-1)} f_{(-m, r)} e^{i m w}\right| \leq M k^{p} r^{2 p}\left(\sum_{n=0} n^{2 p} e^{-n b}\right)^{k-1}=O\left(r^{2 p}\right),
$$


where $M=\sup \left\{\left|f_{m}\right||m|^{-2 p}: m\right.$ in $\left.\mathbb{Z}_{k}\right\}$. Hence, by the equivalence of (A) and (B) in the one variable case, there exists, for each $w$ in $D_{0, k-1}$, a function $H_{w}$, of a single variable, that is holomorphic on $D_{01} \cup I \cup D_{11}$ and can be represented in $D_{01}$ (respectively $D_{11}$ ) by

$$
\sum_{r \geq 0} h_{r}(w) e^{i r z}\left(\text { respectively }-\sum_{r<0} h_{r}(w) e^{i r z}\right),
$$

where

$$
h_{r}(w)=\sum_{(0, k-1)} f_{(-m, r)} \exp (i m w)
$$

By the Hadamard multiplication theorem, for each $w$ in $D_{0, k-1}$

$$
H_{w}^{*}(z)=\sum_{r<0} h_{r}(w) r^{-2 p-2} e^{i r z}
$$

is holomorphic on $D_{11} \cup I$. Each term in (6) is holomorphic on

$$
D_{0, k-1} \times\left(D_{11} \cup I\right)=D_{1 k} \cup\left(D_{0, k-1} \times I\right) .
$$

From (5), for every $y>0$, the series in (6) converges uniformly on the subset of $D_{1 k} \cup\left(D_{0, k-1} \times I\right)$ in which $\operatorname{Im}\left(w_{j}\right) \geq y, j=1, \ldots, k-1$. Hence, by the Weierstrass Theorem on series $\left[3, \quad\right.$ p. 38], $\quad H_{0}^{*}(z)=H_{\left(z_{1}, \ldots, z_{k-1}\right)}^{*}\left(z_{k}\right)$ is holomorphic on $D_{1 k} \cup\left(D_{0, k-1} \times I\right)$ as is

$$
H_{0}(z)=-(-1)^{p+2}\left(\partial^{2 p+2} / \partial z_{k}^{2 p+2}\right) H_{0}^{*}(z)=H_{\left(z_{1}, \ldots, z_{k-1}\right)}\left(z_{k}\right) .
$$

By the same argument, $H_{w}(z)-h_{0}(w)$ and, hence, $H_{0}(z)=H_{\left(z_{1}, \ldots, z_{k-1}\right)}\left(z_{k}\right)$ are holomorphic on $D_{0 k} \cup\left(D_{0, k-1} \times I\right)$. Hence, $H_{0}(z)$ is holomorphic on $D_{0 k} \cup\left(D_{0, k-1} \times I\right) \cup$ $D_{1 k}$. Further, $H_{0}(z)=\hat{f}_{0}(z)$ in $D_{0 k}$ and $H_{0}(z)=-\hat{f}_{1}(z)$ in $D_{1 k}$.

Conversely, suppose for every even integer $c \leq 2^{k}-2$ there exists a function $H_{c}$ holomorphic on $D_{c k} \cup\left(D_{c / 2, k} \times I\right) \cup D_{c+1, k}$ such that $H_{c}(z)=\hat{f}_{c}(z)$ in $D_{c k}, H_{c}(z)=-\hat{f}_{c+1}(z)$ in $D_{c+1, k}$.

Let $y>0$ and $c \leq 2^{k}-2$ be an even integer. Given $z$ in $D_{c k} \cup D_{c+1, k}$, define $z^{\prime}=$ $\left(z_{1}^{\prime}, z_{2}^{\prime}, \ldots, z_{k}^{\prime}\right)$, where, for $j=1, \ldots, k-1$,

$$
z_{j}^{\prime}=z+i y(-1)^{((c, j))}
$$

and $z_{k}^{\prime}=z_{k}$. Further, define $G_{c}(z)=H_{c}\left(z^{\prime}\right)$. Let $V^{*}$ be a complex neighborhood of $I$ whose intersection with the real axis is $I$, and let $V^{* *}$ be the open set in $\mathbb{C}_{k-1}$ that satisfies

$$
\left|\operatorname{Im}\left(z_{\mathrm{j}}\right)\right|<y \quad(j=1, \ldots, k-1) .
$$

Then $V=V^{* *} \times V^{*}$ is a $\mathbb{C}_{k}$ neighborhood of $T_{k-1} \times I$ and $G_{c}(z)$ is holomorphic on $V \cup D_{c k} \cup D_{c+1, k}$. Further,

$$
G_{c}(z)=\sum_{(c, k)} f_{m} e^{-\|m\| y} e^{i m z}
$$


in $D_{c k}$ and

$$
G_{c}(z)=-\sum_{(c+1, k)} f_{m} e^{-\|m m\| y} e^{i m z}
$$

in $D_{c+1, k}$. Hence, by Theorem 1 , for every $g$ in $A_{p k}\left(T_{k-1} \times I\right)$,

$$
\sum f_{m} e^{-\|m\| y} g_{-m}=0 .
$$

Hence, $\langle f, g\rangle=0$.

A. Janusauskas [5] has extended a well-known one-variable theorem to two variables as follows: A necessary and sufficient condition that $\sum_{r \geq 0} \sum_{s \geq 0} a_{r s} z^{r} w^{s}$ be an entire function of $(1-z)^{-1}$ and $(1-w)^{-1}$ is that there exists an entire function, $A(z, w)$, of exponential type zero, such that $A(r, s)=a_{r s}$. With the restriction that the coefficients be polynomially bounded, we may extend Janusauskas's result to $k$ variables.

THEOREM 3. Let $f$ be in $K_{p k}$. The following statements are equivalent:

(i) There exists a function $H$ that is an entire function of the $k$ variables $\left(1-\exp \left(i z_{j}\right)\right)^{-1}$ $(j=1, \ldots, k)$ whose restriction to $D_{c k}$ is $(-1)^{r} f_{c}\left(c=0,1, \ldots, 2^{k}-1\right)$, where $r=\sum_{j=0}^{k}((c, j))$.

(ii) $f$ is null on any open subset of $T_{k}$ that does not contain the origin.

(iii) There exists a polynomial $P$ in $k$ variables such that $P\left(m_{1}, \ldots, m_{k}\right)=f_{m}$ for every $m=\left(m_{1}, \ldots, m_{k}\right)$ in $\mathbb{Z}_{k}$.

Proof. (iii) implies (i) with

$$
H(z)=P\left(\partial / \partial z_{1}, \ldots, \partial / \partial z_{k}\right) \prod_{i=1}^{k}\left(1-\exp \left(-i z_{j}\right)\right)^{-1} .
$$

To show that (i) implies (ii), let $j$ be a fixed positive integer not exceeding $k$. Pair the integers $0, \ldots, 2^{k}-1$ into pairs $\left(c_{1}, c_{2}\right)$, where $c_{1}$ and $c_{2}$ have binary expansions that differ in and only in the coefficient of $2^{k-j}$ and $\sum_{t=1}^{k}\left(\left(c_{1}, t\right)\right)$ is even. Let

$$
a=\sum_{i=1}^{j-1}\left(\left(c_{1}, t\right)\right) 2^{j-1-2}
$$

and

$$
b=\sum_{t=j+1}^{k}\left(\left(c_{1}, t\right)\right) 2^{k-t} .
$$

In Theorem 2, we considered the notationally simplest case with $j=k, a= \pm c_{1} / 2$ and $b$ an empty sum. Hence, by rearranging the variables to fit the statement of Theorem 2 , we have that since, for each pair $\left(c_{1}, c_{2}\right), H$ is represented in $D_{c_{1} k}$ by $\hat{f}_{c_{1}}$ and $H$ is represented 
in $D_{c_{2} k}$ by $-\hat{f}_{c_{2}}$ and $H$ is holomorphic on

$$
D_{c_{1} k} \cup\left(D_{a, j-1} \times(0,2 \pi) \times D_{b, k-j}\right) \cup D_{c_{2} k},
$$

then $f$ is null on $T_{j-1} \times(0,2 \pi) \times T_{k-j}$.

If $f$ is null on open sets $E$ and $G$, then $f$ is null on their union. For, if $g$ is in $A_{p k}(E \cup G)$, then with a proper choice of partitions of unity on the support of $g$, we may write $g$ as a sum of a member of $A_{p k}(E)$ and a member of $A_{p k}(G)$. Therefore, since the union of $T_{j-1} \times(0,2 \pi) \times T_{k-j}(j=1, \ldots, k)$ is $T_{k}-\{0\}, f$ is null on any open set that does not contain the origin.

To see that (ii) implies (iii), let $Q$ be a polynomial in one variable such that $Q(0)=0$, $Q(1)=1$ and

$$
Q^{(v)}(0)=Q^{(v)}(1)=0 \quad(v=1, \ldots, 2 p+k+3) .
$$

For every $\delta>0$, define

$$
V_{\delta}(t)=\left\{\begin{array}{lll}
0 & \text { if } & |t|<\delta \\
Q\left(\delta^{-1} \cdot(|t|-\delta)\right) & \text { if } & \delta \leq|t| \leq 2 \delta \\
1 & \text { if } & 2 \delta<|t| \leq \pi
\end{array}\right.
$$

For every $x$ in $T_{k}$, let $H_{\delta}(x)=\prod_{j=1}^{k}\left(1-V_{\delta}\left(x_{j}\right)\right)$.

Let $\sum c_{m} e^{i m x}$ be the Fourier series for $H_{\delta}$. Let $m$ have $s(0 \leq s \leq k-1)$ zero coordinates. Then for all $m$,

$$
\begin{aligned}
c_{m}= & (2 \pi)^{-k} \sum_{a=1}^{k}(-1)^{a} \sum_{*} \Pi\left(\int_{-\pi}^{\pi} V_{\delta}(x) \exp \left(-i m_{u} x\right) d x\right) \\
& \times \prod\left(\int_{-\pi}^{\pi} \exp \left(-i m_{u} x\right) d x\right),
\end{aligned}
$$

where $\sum_{*}$ is the sum over all integer sets $\left(r_{1}, \ldots, r_{q}\right)$ with $1 \leq r_{1}<\ldots<r_{q} \leq k$, the first product is taken over all $m_{u}$ for which $u=r_{j}$ for some $j \leq q$, and the second product is taken over the remaining $m_{u}$. Since any term, for which a non-zero $m_{u}$ appears in the second product, vanishes, we may write

$$
\left|c_{m}\right| \leq \sum_{u=0}^{s}(2 \pi)^{u-k}\left(\int_{-\pi}^{\pi}\left|V_{\delta}^{(2 p+2)}(x)\right| d x\right)^{k-u}\left(\int_{-\pi}^{\pi}|V(x)| d x\right)^{u} \cdot\left(\prod_{m_{j} \neq 0}\left|m_{j}\right|^{2 p+2}\right)^{-1} .
$$

Hence, for some constant $B>0$,

$$
\sum_{|m| \neq 0}|m|^{2 p}\left|c_{m}\right| \leq B \sum_{s=0}^{k-1}\left(\begin{array}{l}
k \\
s
\end{array}\right)(k-s)^{2 p}\left(\sum_{n \neq 0}|n|^{-2}\right)^{k-s}
$$

Therefore, $H_{\delta}$ is in $A_{p k}$. 
Now, let

$$
g(x)=\prod_{r=1}^{k}\left(1-\exp \left(-i x_{r}\right)\right)^{v(r)}
$$

where $v(1), \ldots, v(k)$ are non-negative integers whose sum is greater than $2 p+2 q$, where $2 q$ is the smallest even integer not less than $(k+2) / 2$. Here $g\left(1-H_{\delta}\right)$ has support in $T_{k}-\{0\}$. Let $\left\{b_{m}^{\delta}\right\}$ be the sequence of Fourier coefficients of $g H_{\delta}$. Then

$$
\left\|g-g\left(1-H_{\delta}\right)\right\|_{2 p}=\max \left\{\left|b_{0}^{\delta}\right|, \Sigma^{\prime}|m|^{2 p}\left|b_{m}^{\delta}\right|\right\} \text {. }
$$

Since $g(x) H_{\delta}(x)$ converges pointwise to zero and on $T_{k}$ is uniformly bounded by $2^{v}\left(\max _{0 \leq t \leq 1}|1-Q(t)|\right)^{k}$, we have that $b_{0}^{\delta} \rightarrow 0$ as $\delta \rightarrow 0$.

By the Cauchy-Schwarz inequality,

But

$$
\sum^{\prime}|m|^{2 p}\left|b_{m}^{\delta}\right| \leq\left(\sum^{\prime}|m|^{-4 q}\right)^{1 / 2}\left(\sum^{\prime}\left|b_{m}^{\delta}\right|^{2}|m|^{4 p+4 q}\right)^{1 / 2}
$$

$$
\sum^{\prime}|m|^{-4 a}=k+\sum_{j=2}^{\infty}\left(\sum_{j-1<|m| \leq j}|m|^{-4 a}\right)=k+\sum_{j=2}^{\infty} O\left(j^{-2}\right) .
$$

Further,

$$
\sum^{\prime}\left(|m|^{2 p+2 q}\left|b_{m}^{\delta}\right|\right)^{2}=(2 \pi)^{-k} \int_{T_{k}}\left|\Delta^{p+a} g(x) H_{\delta}(x)\right|^{2} d x .
$$

The Laplacian of order $p+q$ in (7) is a finite linear combination of terms of the form

$$
\prod_{r=1}^{k} D^{2 u(r)}\left(1-\exp \left(-i x_{r}\right)\right)^{v(r)}\left(1-V_{\delta}\left(x_{r}\right)\right)
$$

where $u(1)+\ldots+u(k)=p+q$. Ignore for a moment the index $r$. Let $L+M=2 u$; the derivative of order $L$ of $\left(1-V_{\delta}(x)\right)$ vanishes if $x>2 \delta$ and is $O\left(\delta^{-L}\right)$ if $x \leq 2 \delta$. The derivative of order $M$ of $(1-\exp (-i x))^{v}$ is $O\left(x^{j}\right)$ where $j=\max (0, v-M)$. Each factor in (8) is, therefore, $O\left(\delta^{h}\right)$ where $h=j-L \geq v-2 u$. Since

$$
\sum_{r=1}^{k}(v(r)-2 u(r))>0
$$

the integral in (7) is $O(1)$ as $\delta \rightarrow 0$. Hence, $g\left(1-H_{\delta}\right)$ converges to $g$ in the $\|\cdot\|_{2 p}$ norm.

Hence, by (1), for every $n$ in $\mathbb{Z}_{k}, \sum f_{m} g_{n-m}=0$, where $g_{m}$ is the $m$ th Fourier coefficient of $g$ and is equal to $(-1)^{\|m\|}\left(\begin{array}{c}0 \\ m\end{array}\right)$, where $\left(\begin{array}{l}v \\ m\end{array}\right)$ is the product of the binomial coefficients $\left(\begin{array}{c}v(r) \\ m_{r}\end{array}\right)(r=1, \ldots, k)$ provided $0 \leq-m_{r} \leq v(r)$ for each $r$, and $g_{m}=0$ otherwise. Therefore, for every $n$ in $\mathbb{Z}$,

$$
\sum_{0 \leq m_{r} \leq v(r)} f_{n+m}(-1)^{\|m\|}\left(\begin{array}{c}
v \\
m
\end{array}\right)=0 .
$$


That is, the application of any finite difference operator of total order greater than $2 p+2 q$ annihilates $f$. Thus, $f$ is given by a polynomial.

3. Statements (B) and (C). For $k \geq 2$, we consider the formal expression:

$$
F(x)=f_{0}\left(x_{1}+\ldots+x_{k}\right)^{2 D+2} a(p, k)+(-1)^{p+1} \sum^{\prime} f_{m}|m|^{-2 p-2} e^{i m x},
$$

where $a(p, k)=\left((2 p+2) ! k^{p+1}\right)^{-1}$. We can consider $F$ in a distribution sense; that is, for any $c(x)=\sum c_{m} e^{i m x}$ that is $\mathscr{C}^{\infty}$ on $T_{k}$,

$$
\begin{aligned}
F(c)= & (2 \pi)^{-k} \int_{T_{k}} a(p, k) f_{0}\left(x_{1}+\ldots+x_{k}\right)^{2 p+2} c(x) d x \\
& +(-1)^{p+1} \sum^{\prime} f_{m} c_{-m}|m|^{-2 p-2} .
\end{aligned}
$$

THEOREM 4. Let $f$ be in $K_{\mathrm{pk}}$. If $f$ is null on an open set $E$ in $T_{k}$, then the distribution $\triangle^{\mathrm{p}} F \mid E$ is defined by a harmonic function. Further, if the series in (9) is the Fourier series for an $L_{1}$ function, $F^{*}$, then $f$ is null on any open set on which $F$ has a vanishing Laplacian of order $p+1$.

Proof. Let $f$ be null on $E, c$ be in $\mathscr{C}^{\infty}(E)$ and $F^{*}$ be the distribution defined by the series in (9). Then

$$
\begin{aligned}
\Delta^{\mathrm{p}} F(\Delta c) & =\Delta^{\mathrm{p}}\left(F-F^{*}\right)(\Delta c)+\Delta^{p} F^{*}(\Delta c) \\
& =\Delta^{\mathrm{p}+1}\left(F-F^{*}\right)(c)+F^{*}\left(\Delta^{\mathrm{p}+1} c\right) \\
& =f_{0} c_{0}+\sum^{\prime} f_{m} c_{-m}=0 .
\end{aligned}
$$

By $\left[4\right.$, p. 3], $\Delta^{p} F \mid E$ is defined by a harmonic function.

Let $F^{*}$ be in $L_{1}$ and suppose $\Delta^{p+1} F(x)=0$ for every $x$ in $E$. Let $g$ be in $A_{p k}(E)$. By considering partitions of unity whose supports are open balls covering the support of $g$, we may restrict our attention to $g$ having support in an open ball, $B(t, r)$, of radius $r$ and center $t$ which we may take as the origin. Then $\breve{g}(x)=g(-x)$ also has support in $B(0, r)$.

Let

$$
H(x)=(2 \pi)^{-k} \int_{T_{k}} F^{*}(x-u) \check{g}(u) d u .
$$

Since $\sum f_{m} g_{-m}$ converges absolutely,

But

$$
\sum_{m=1}^{k} f_{m} g_{-m}=\Delta^{p+1} H(0)
$$

$$
\begin{aligned}
\Delta^{p+1} H(0) & =-(2 \pi)^{-k} \int_{B(0, r)} a(p, k) f_{0} \check{g}(u) \Delta^{p+1}\left(u_{1}+\ldots+u_{k}\right)^{2 \mathrm{p}+2} d u \\
& =-f_{0} g_{0} .
\end{aligned}
$$

Hence, $\langle f, g\rangle=0$. 
Let $G(x)$ be the Green's function on $T_{k}$; that is, $G(x)$ is the $L_{1}$ function whose Fourier series is $\sum^{\prime}|m|^{-2} e^{i m x}[9]$.

Corollary. Let $f$ be $K_{0 k}$ and $F$ be given by (9). Let $x_{0}$ be in $T_{k}$ and $E$ be an open neighborhood of $x_{0}$. If $f$ is null on $E-\left\{x_{0}\right\}$, then there exists a number $z$ such that

$$
F(x)-z\left(x_{1}+\ldots+x_{k}\right)^{2} /(2 k)-z G\left(x-x_{0}\right)
$$

is harmonic on $E$.

Proof. We need only show that for some number $z$ the multisequence $\left\{f_{m}-\right.$ $\left.z\left(\exp \left(i m x_{0}\right)\right)\right\}$ is null on $E$. Let us assume $x_{0}=0$. In the terminology of [6], $K_{0 k}$ is $L_{\infty}\left(\mathbb{Z}_{k}\right)$; and, by identifying a function with the sequence of its Fourier coefficients, we may equate $A_{0 k}$ with $L_{1}\left(\mathbb{Z}_{k}\right)$.

Let $I$ be the closed ideal in $\mathrm{L}_{1}\left(\mathbb{Z}_{\mathrm{k}}\right)$ consisting of all $g$ such that $f^{*} g=0$; the zero-set, $Z(I)$, is contained in $E^{c}-\{0\}$. Let $E^{\prime}$ be any open neighborhood of 0 whose closure is contained in $E$. Let $g$ and $h$ be in $A_{0 k}\left(E^{\prime}\right)$ with $g(0)=h(0)=1$. By [6, 7.2.4], we have that $h-g$ is in $I$. Therefore, $\langle f, h-g\rangle=0$. Hence, choose any $g$ in $A_{0 k}\left(E^{\prime}\right)$ with $g(0)=1$ and define $z=\langle f, g\rangle$. The multisequence $\left\{f_{m}-z\right\}$ is null on $E^{\prime}$ and on $E-\{0\}$ and, hence, on $E$ their union.

4. Diagonal Subsets of $T_{k}$. Given $k \geq 2$ and $d=\left(d_{1}, \ldots, d_{k}\right)$ a $k$-tuple of unit integers, $d_{i}= \pm 1$, define, for any $E \subset T_{k-1}$,

$$
E(d)=\left\{x \text { in } T_{k}:\left(d_{1} x_{1}-d_{k} x_{k}, \ldots, d_{k-1} x_{k-1}-d_{k} x_{k}\right) \text { is in } E\right\} .
$$

We may picture in $T_{2}$ a subset of $T_{1}$ projected diagonally. We note that a necessary and sufficient condition that a subset $S$ of $T_{k}$ be $\bar{E}(d)$ for some $E \subset T_{k-1}$ is that, for every $t$ in $T_{1}, S+t d=S$.

Let $\sum g_{m}\left(m \in \mathbb{Z}_{k}\right)$ be an absolutely convergent series and let $g(x)=\sum g_{m} e^{i m x}\left(x\right.$ in $\left.T_{k}\right)$. For any integer $h$, define the sequence $\left\{g_{n}^{h}\right\}\left(n \in \mathbb{Z}_{k-1}\right)$ by

$$
g_{n_{1}, \ldots, n_{k-1}}^{h}=g_{d_{1} n_{1}, \ldots, d_{k-1} n_{k-1}, d_{k}(h-s(n))}
$$

where $s(n)=n_{1}+\ldots+n_{k-1}$. Also define for all $y$ in $T_{k-1}$, the function $g_{h}(y)=$ $\sum g_{n}^{h} e^{i n y}\left(n \in \mathbb{Z}_{k-1}\right)$.

Lemma 1. Let $S$ be an open subset of $T_{k}$ such that $S=E(d)$ for some $E \subset T_{k-1}$ and some $k$-tuple $d$ of unit integers. A necessary and sufficient condition for the function $g$, given above, to vanish at every point not in $S$ is that, for every integer $h, g_{h}(y)=0$ for every y not in $E$. If $g$ has support in $S$, each $g_{h}$ has support in $E$. If there exists a compact subset $K \subset E$ such that, for every $h, g_{h}$ has support in $K$, then the support of $g$ is in $S$.

Proof. If $y$ is not in $E$, then, for every $t$ in $T_{1}$,

$$
x=\left(d_{1}\left(y_{1}+t\right), \ldots, d_{k-1}\left(y_{k-1}+t\right), d_{k} t\right)
$$


is not in $S$, and, hence, $g(x)=0$. But if $x$ is in the form (11),

$$
g(x)=\sum_{h=-\infty}^{\infty} g_{h}(y) e^{i h t} ;
$$

hence, by Riemann's theorem, $g_{h}(y)=0$ for all $h$.

Conversely, since every $x$ not in $S$ is representable in the form (11) for some $t$ in $T_{1}$ and $y$ not in $E$, if $g_{h}(y)=0$ for every $y$ not in $E, g(x)=0$ for every $x$ not in $S$. The remaining statements of the lemma follow immediately.

Let $f$ be in $K_{p k}$ and, for every integer $h$ define the sequence $f^{h}=\left\{f_{n}^{h}\right\}\left(n \in \mathbb{Z}_{k-1}\right)$ as in (10). For convenience, let us consider the $(k-1)$-tuple, $n$, as a lattice point on the hyperplane

$$
d_{1} x_{1}+\ldots+d_{k} x_{k}=h
$$

and the norm, $|n|$, as the distance from $n$ to the point

$$
\left(d_{1} h / k, d_{2} h / k, \ldots, d_{j} h / k\right) \text {. }
$$

Hence

$$
\left|f_{n}^{h}\right||n|^{-2 p}=\left|f_{m}\right|\left(|m|-h^{2} / k^{2}\right)^{-p}=\left|f_{m}\right||m|^{-2 p} O(1),
$$

where $m$ and $n$ are related by (10). Thus, if $f$ is in $K_{p k}, f^{h}$ is in $K_{p, k-1}$ for every $h$. Further, if the sequence $\left\{\left|f_{n}^{h}\right|\left(|n|^{2}+h^{2}\right)^{-p}\right\}$ is uniformly bounded with respect to $h$, then $f$ is in $K_{p k}$.

THEOREM 5. Let $S$ be an open subset of $T_{k}$ such that $S=E(d)$ for some open set $E$ in $T_{k-1}$ and for some $k$-tuple $d$ of unit integers. Let $f$ be in $K_{p k}$, and $f^{h}$ be as defined above. A necessary and sufficient condition that $f$ be null on $S$ is that $f^{h}$ is null on $E$ for all integers $h$.

Proof. Suppose $f$ is null on $S$. Let $h^{\prime}$ be an integer and

$$
q(y)=\sum q_{n} e^{i n y} \quad\left(n \in \mathbb{Z}_{k-1}, y \in T_{k-1}\right)
$$

be in $A_{p, k-1}(E)$. For every $m$ in $\mathbb{Z}_{k}$, define $g_{m}=q_{d_{1} n_{1} \ldots, d_{k-1} n_{k-1}}$ if $m . d=-h^{\prime}$ and $g_{m}=0$ otherwise. Let us define $g_{n}^{h}\left(n \in \mathbb{Z}_{k-1}, h \in \mathbb{Z}\right)$ by $(10)$. Then $g_{n}^{h}=0$ if $h \neq h^{\prime}$ and $g_{n}^{h^{\prime}}=q_{n}$. Thus $g_{h^{\prime}}(y)=q(y)$ and $g_{h}(y)=0$ if $h \neq h^{\prime}$. The support of each $g_{h}$ is contained in the support of $q$; therefore, by Lemma 1 , the support of $g$ is in $S$. Further,

$$
\sum|m|^{2 p}\left|g_{m}\right|=O\left(|n|^{2 p}\right)\left|q_{n}\right| ;
$$

thus $g$ is in $A_{p k}(S)$ and $\langle f, g\rangle=0$. But

$$
\langle f, g\rangle=\sum f_{n}^{h^{\prime}} q_{-n}=\left\langle f^{h^{\prime}}, q\right\rangle .
$$

Thus, $f^{h^{\prime}}$ is null on $E$.

Conversely, if $f^{h}$ is null on $E$ for all $h$ and $g$ is in $A_{p k}(S)$, then

$$
\langle f, g\rangle=\sum_{h=-\infty}^{\infty} \sum_{\mathbf{Z}_{k-1}} f_{n}^{h} g_{-n}^{-h}=\sum_{h=-\infty}^{\infty}\left\langle f^{h}, g_{-h}\right\rangle .
$$

By Lemma $1, g_{-h}$ is in $A_{p, k-1}(E)$; thus $\langle f, g\rangle=0$. 
Corollary. Let $f$ be in $K_{\mathrm{pk}}$. A necessary and sufficient condition that $f$ be null on any open subset of $T_{k}$ that does not include the line $L$ given by $d_{1} x_{1}=d_{2} x_{2}=\ldots=d_{k} x_{k}, d_{j}= \pm 1$, $1 \leq j \leq k$, is that for every integer $h$ the sequence $\left\{f_{n}^{h}\right\}\left(n \in \mathbb{Z}_{k-1}\right)$ formed from $f$ as in (10), satisfy statements (i), (ii) and (iii) of Theorem 3 with $k$ replaced by $k-1$. If $k=2$ and I is an open interval in $T_{1}$ containing the origin, then a necessary and sufficient condition that $f$ be null on $I\left(\left(d_{1}, d_{2}\right)\right)-L$ is that, for every integer $h$, there exists a one-variable function that is analytic on I except for a pole of order at most $2 p+1$ at the origin and representable in the upper and lower half planes respectively by

$$
\sum_{n \geq 0} f_{d_{1} n, d_{2}(h-n)} e^{i n z} \text { and } \quad-\sum_{n<0} f_{d_{1} n, d_{2}(h-n)} e^{i n z} .
$$

Proof. By Theorem 5, if $f$ is null of $T_{k}-L$, statement (ii) of Theorem 3 follows.

By Theorem 5, $f$ is null on $I\left(\left(d_{1}, d_{2}\right)\right)-L$ if and only if $f^{h}$ is null on $I-\{0\}$ for every $h$; but, in the one-variable case, this is equivalent to the functions in (12) being analytic continuations of each other across the components of $I-\{0\}$, which, for each $h$, is equivalent, by the theorem of S. M. Shah [7], to the existence of a pole of order at most $2 p+1$ at the origin.

\section{REFERENCES}

1. A. Beurling, Analytic continuation across a linear boundary, Acta Math. 128 (1972), 153-182.

2. F. W. Carroll and D. J. Troy, Distributions and analytic continuation, J. Analyse Math. XXIV (1971), 87-100.

3. B. A. Fuks, Analytic functions of several variables (American Mathematical Society, Providence, R.I., 1963).

4. M. Herve, Analytic and plurisubharmonic functions, Lecture Notes in Mathematics 198 (Springer-Verlag, 1971).

5. A. Janusauskas, Analytic continuation of sums of double power series. Differencial'nye Uravnenija i Primenen.-Trudy Sem. Processy Optimal. Upravlenija I Sekcija, 18 (1977), 51-73.

6. W. Rudin, Fourier analysis on groups (Interscience Publications, Wiley, 1962).

7. W. Rudin, Lectures on the edge-of-the-wedge theorem (American Mathematical Society, Providence, R.I., 1971).

8. S. M. Shah, On the singularities of a class of functions on the unit circle, Bull. Amer. Math. Soc., 52 (1946), 1053-1056.

9. V. Shapiro, Generalized laplacians, Amer. J. Math. 78 (1956), 497-508.

Purdue UniverstTy

Calumet Campus

HAMMOND

INDIANA 46323 